\title{
HemiCAP-Wave ${ }^{\circledR}$ Implant in Salvage Procedure of a Large Trochlea Lesion in the Knee in a 37 Year Old Former Active Man
}

Jens Ole Laursen*

Department of Orthopaedic and Emergency Hospital of South Jutland, Denmark

\begin{abstract}
We present a case report using this inlay resurfacing prosthesis as a salvage procedure in a 37 year old man with a large trochlea defect after 6 former cartilage procedures. The early follow-up results after treatment with this customized metal mini-prosthesis in a challenging young active patient with a focal (osteo) chondral lesions and a history of failed previous cartilage surgery demonstrated significant pain and subjective outcome improvements at 4 years.
\end{abstract}

Keywords: Knee; Cartilage defects; Salvage procedure-Osteoarthritis; Resurfacing arthroplasty

\section{Introduction}

In 2009, a large anatomic metallic implant for Trochlea resurfacing (HemiCAP-Wave Focal Trochlea Condyle Resurfacing Arthroplasty, Arthrosurface Inc, Franklin, MA, USA) was introduced for treatment of larger full thickness trochlea lesions or isolated OA in the patellofemoral joint. The arthroplasty intends to replicate the complex contours and biomechanics of the joint space by mapping this intraoperatively [14] (Figure 1). We present a case report using this inlay resurfacing prosthesis as a salvage procedure in a 37 year old man with a large trochlea defect after 6 former cartilage procedures.

\section{Device description}

The HemiCAP-Wave resurfacing implant consists of two components: fixation and modular articular components, the two connected by a morse taper. (HemiCAP-Wave Resurfacing Arthroplasty, Arthrosurface Inc, Franklin, MA, USA) The fixation component is a titanium cancellous screw with full- length cannulation. The cobalt chrome articular component is available in $35 \times 35 \mathrm{~mm}$ diameter and comes in eight different offset configurations corresponding to the superior/inferior and medial/lateral radius of curvatures at the implant

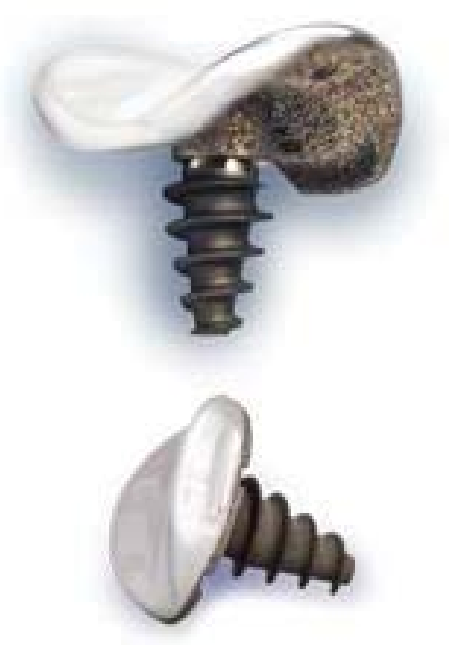

Figure 1: HemiCAP-wave. site (Figure 1) which thereby tends to replicate the contours of this complex joint. A polyethylene $30 \mathrm{~mm}$ patella button was available if needed.

\section{Surgical Procedure}

The procedure was initiated with a standard arthroscopy to identify cartilage status and treat any concomitant intra-articular pathology. The defect was exposed using a small para-patellar incision and the cartilage lesion was sized. Sizing trials were used to confirm an accurate fit to the surrounding cartilage, and proper match the individual contours of the knee (inlay prosthesis), making it more custom-made than previous seen on lay prosthesis. The resurfacing implant was fixed press-fit onto the fixation screw and seated flush or slightly recessed to the surrounding articular cartilage surface. A standardized rehabilitation protocol with free range of motion was allowed immediately after the operation.

\section{Outcome evaluation}

The patient were assessed PO, after 3 months, 1, 2 and 4 years by American Knee Society Scores (AKSS) clinical and function-subscales [5] and a pain score using a numerical ranking scale ( $0-10), 10$ being the worst possible pain.

\section{Radiographic evaluation}

The osteoarthritis (OA) grade at standard weight-bearing AP, axial and patellae-skyline radiographs was evaluated using the KellgrenLawrence scale (KL) [6] for both medial and lateral tibiofemoral compartments and the patellofemoral compartment preoperatively and at the clinical follow-up to investigate $34 \mathrm{OA}$ development after surgery $[7,8]$.

\section{Case Presentation}

A 37 year old physiotherapist with a soccer injury to his left knee

*Corresponding author: Jens Ole Laursen, Department of Orthopaedic and
Emergency Hospital of South Jutland, Denmark, Tel: 0045-29173433; E-mail: Jens.Ole.Laursen@rsyd.dk

Received March 15, 2017; Accepted April 03, 2017; Published April 10, 2017

Citation: Laursen JO (2017) HemiCAP-Wave $\AA$ Implant in Salvage Procedure of a Large Trochlea Lesion in the Knee in a 37 Year Old Former Active Man. J Tissue Sci Eng 8: 198. doi: 10.4172/2157-7552.1000198

Copyright: $\odot 2017$ Laursen JO. This is an open-access article distributed under the terms of the Creative Commons Attribution License, which permits unrestricted use, distribution, and reproduction in any medium, provided the original author and source are credited. 
Citation: Laursen JO (2017) HemiCAP-Wave $®$ Implant in Salvage Procedure of a Large Trochlea Lesion in the Knee in a 37 Year Old Former Active Man. J Tissue Sci Eng 8: 198. doi: 10.4172/2157-7552.1000198

Page 2 of 3

in 2010 leaving constant pain and disability. Arthroscopy reveals a smaller cartilage lesion in trochlea and a micro-fracture procedure was done without sufficient effect. A new procedure was done, still without effect, and next step was auto-grafting with True- fit plugs from the rim of trochlea - but failure only made the lesion more profound - and CT and MR-scans confirms this (Figure 2) with lesions at $22 \times 15 \mathrm{~mm}$ in diameter and $15 \mathrm{~mm}$ deep - he was now scheduled for autologous spongiosa-plastic covered with a periostflap. There was no effect of this operation, and a new arthroscopy found progression of the lesions in trochlea (Figure 3) and he was now remitted to operation with the HemiCAP-Wave-resurfacing prosthesis, which was done 3 years after his index injury and 6 former arthroscopies and operations. He had more or less been out of work in this period as a physio-therapist. He used daily painkillers and had a low AKSS-score (Table 1). Postoperatively he gained his function back and was fulltime working again after 3 months, and still at 4 years control he is full-time working, using no painkillers and can run and cycle without problems. He was controlled regularly at clinical and radiological controls at 3 months, 1, 2 and 4 years and he remained almost pain-free in the operated knee. VAS preoperative at 8 fell to 1-2 at the present and AKSS rising from 45 postoperative and at 4 years at 95 . KL-grade was preoperative $0-1$ in all compartments, and now at 4 years, 1-2 in all compartments. There were no signs of any loosening of the HemicapWave" (Figure 4).

\section{Conclusion}

The early follow-up results after treatment with this customized metal mini-prosthesis in a challenging young active patient with a focal (osteo) chondral lesions and a history of failed previous cartilage surgery demonstrated significant pain and subjective outcome improvements at 4 years. The advantage of this large inlay prosthesis is the possibility to restore the contours of this complex patella-femoral joint and thereby the dynamics. The patient had been out of work as a physiotherapist for almost 3 years and was at risk of losing his ability to work completely and thereby becoming a heavy financial burden on society. We know from other studies [7-10] that the loss of working ability can be fatal for such patients, who are still of working age, and the economic costs for society enormous, especially for these younger patients in the "Treatment

\begin{tabular}{|l|c|c|c|c|c|}
\hline & Preop & 3 months & 1 year & 2 years & 4 years \\
\hline Pain score - VAS & 8 & 4 & 2 & 1 & 1 \\
\hline AKSS func. & 50 & 80 & 95 & 95 & 95 \\
\hline AKSS obj. & 45 & 85 & 90 & 90 & 95 \\
\hline KL-grade & 1 & 1 & 1 & 1 & 1 \\
\hline Medial & 0 & 0 & 0 & 1 & 1 \\
\hline Lateral & 1 & 1 & 1 & 1 & 2 \\
\hline Patello/femoral & & & & & \\
\hline
\end{tabular}

Table 1: Clinical and radiographic scores preoperative and at follow-up.
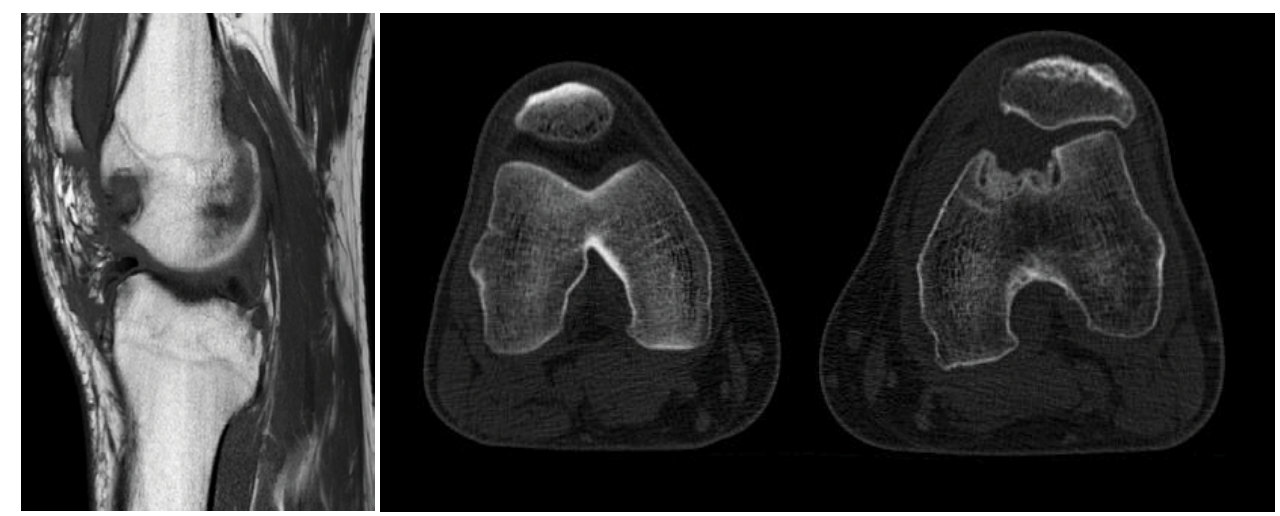

Figure 2: MR and CT scans with osteochondral defect in trochlea (incl. normal contralateral knee).
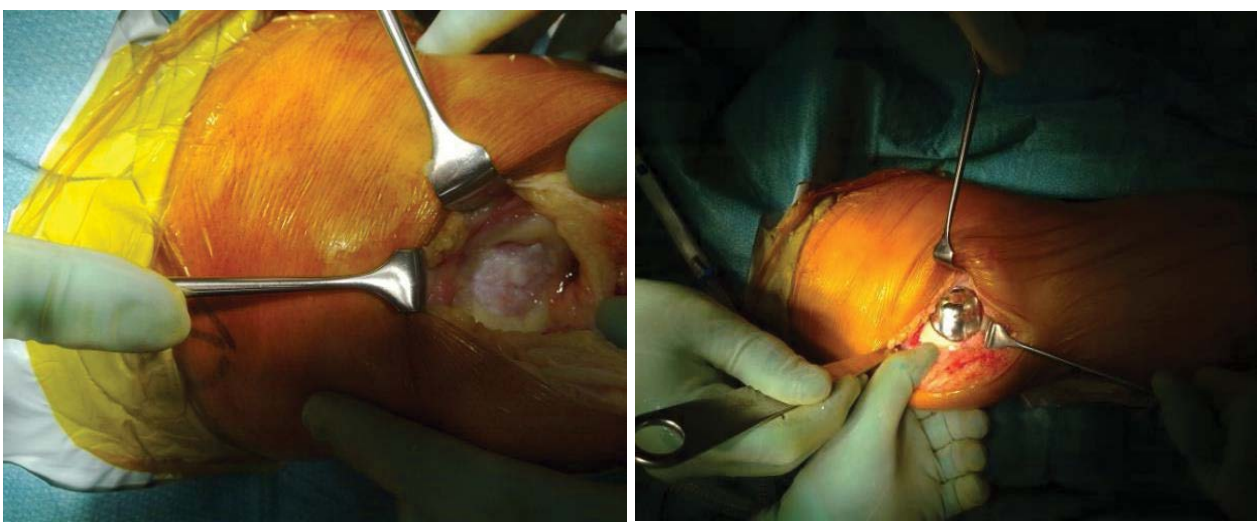

Figure 3: Cartilage defect in trochlea and HemiCAP-wave in place. 


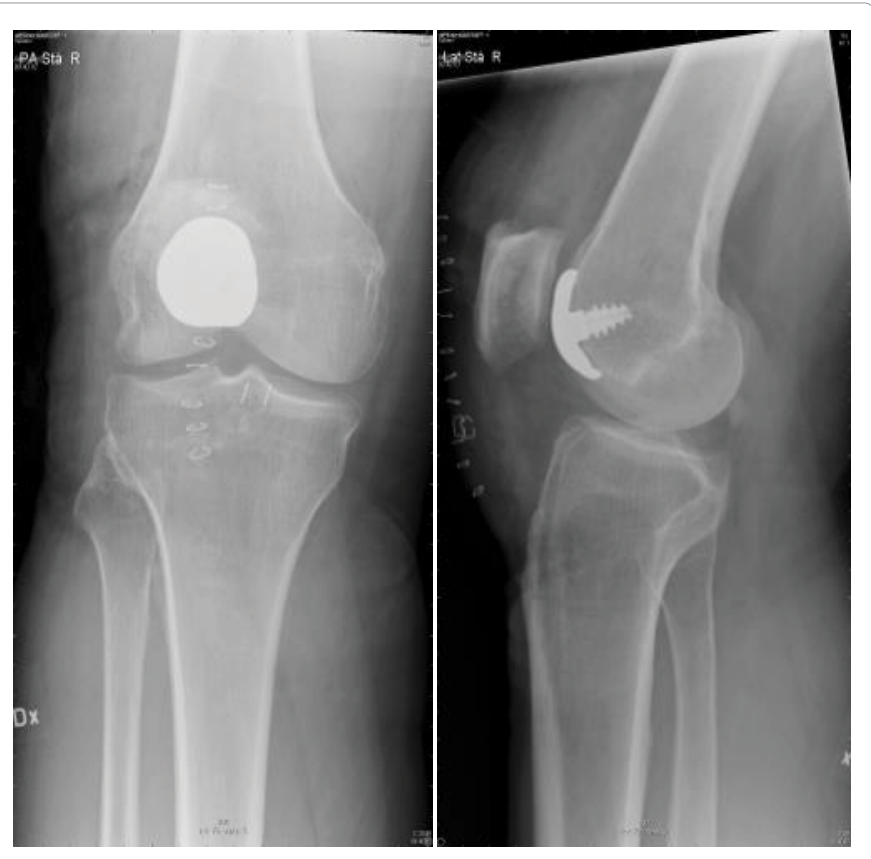

Figure 4: Radiographs post-operative.

Gap", meaning patients between 35 and 60 years, where cartilage procedures such as ACI (autologous chondrocyte implantation, True Fit or microfracture technique is dubious, and jet not old or worn enough for a TKA. It is estimated that only in the US, 3.6 million patients with symptomatic knee-pain fall into this group [10].

\section{References}

1. Dhollander AAM, Almquist KF, Moens K, Vandekerckhove PJ, Verdonk R, et al. (2015) The use of a prosthetic inlay resurfacing as a salvage procedure for a failed cartilage repair. Knee Surg Sports Traumatol Arthrosc 23: 2208-2212.

2. Imhoff AB, Feucht MJ, Meidinger G, Schötte PB, Cotic M (2015) Prospective evaluation of anatomic patellofemoral inlay resurfacing: Clinical, radiographic and sports-related results after 24 months. Knee Surg Sports Traumatol Arthrosc 23: 1299-1307.

3. Feucht MJ, Cotic M, Beitzel K, Baldini JF, Meidinger G, et al. (2015) A matchedpair comparison of inlay and onlay trochlear designs for patellofemoral arthroplasty: No differences in clinical outcome but less progression of osteoarthritis with inlay designs. Knee Surg Sports Traumatol Arthrosc.

4. Laursen JO (2016) High mid-term revision rate after treatment of large, full-thickness cartilage lesions and OA in the patellofemoral joint using a large inlay resurfacing prosthesis: HemiCAP-Wave ${ }^{\circledR}$. Knee Surg Sports Traumatol Arthrosc, pp: 1-6.

5. Insall JN, Dorr LD, Scott RD, Scott WN (1989) Rationale of the knee society clinical rating system. Clin Orthop Relat Res 248: 13-14.

6. Kellgren JH, Lawrence JS (1957) Radiological assessment of osteo-arthrosis Ann Rheum Dis 16: 494-502.

7. Laursen JO, Lind M (2015) Treatment of full-thickness femoral cartilage lesions using condyle resurfacing prosthesis. Knee Surg Sports Traumatol Arthrosc 25: 746-751.

8. Laursen JO (2016) Treatment of full-thickness cartilage lesions and early OA using large condyle resurfacing prosthesis: UniCAP. Knee Surg Sports Traumatol Arthrosc 24: 169-1671.

9. Li CS, Karlsson J, Winemaker M, Sancheti P, Bhandari M (2014) Orthopedic surgeons feel that there is a treatment gap in management of early OA: International survey. Knee Surg Sports Traumatol Arthrosc 22: 363-378.

10. London NJ, Miller LE, Block JE (2011) Clinical and economic consequences of the treatment gap in knee osteoarthritis management. Med Hypotheses 76: 887-892. 